\title{
COMPETENCIAS CLAVES PARA LA GESTIÓN EMPRESARIAL DE LAS MYPES
}

Mg. Robert Miranda Castillo (*)

E-mail: rmirandac@unmsm.edu.pe

\begin{abstract}
RESUMEN
Las Micro y Pequeñas Empresas (MYPES) representan el 98\% del total de empresas e iniciativas económicas en el Perú; generan aproximadamente el $75 \%$ del empleo y aportan alrededor del $44 \%$ de la riqueza generada. Las MYPEs exportadoras suman 1,674 de un total de 3,057 empresas, pero por el tamaño de sus ventas su participación es poco significativa, con una participación en las exportaciones de poco menos del 1\%.

La problemática de las MYPEs demanda romper los paradigmas con los que han venido operando por lo que queremos, dado el nuevo contexto, comprender su naturaleza, establecer nuevos retos y valorar su potencial, a fin de establecer nuevas estrategias, pertinentes y generadoras de valor con responsabilidad social.

El eje central de las propuestas debe partir del desarrollo del potencial y capital humano; desarrollar capacidades gerenciales y alianzas estratégicas; incorporar y adaptar tecnología y sistemas de información y comunicaciones; identificar y hacer prospectiva de los mercados, y desplegar una estructura de soporte por parte del Estado, gobiernos nacional y local, teniendo a la propiedad intelectual e industrial como uno de los sistemas de apoyo al desarrollo empresarial.
\end{abstract}

Palabras Clave: MYPES, participación en el mercado, responsabilidad social, prospectiva de mercado.

\section{ABSTRACT}

Micro and small companies represent approximately a $98 \%$ of the total amount of enterprises in Peru by generating by the $75 \%$ of employment and contribute round $44 \%$ of the enterprise generated income revenue. Export micro and small Companies imply 1674 ones from a total amount of 3057 companies however because of their sales size its market participation is a little meaningful and having export participation by less than a $1 \%$.

It's a must to assess, understand both challenges and nature when to micro and small Companies demands to break some paradigms are concerned to settle new pertaining and value generating strategies by considering social responsibility.

Main axis on purposes from human potential and development to develop management capacities, identify and perform market prospective and display a state, local governments support structure having intellectual and industrial rights as one of the system supports on the business development.

Key words: Micro and small Companies, Market participation, Social responsibility, Market prospective .

* Licenciado en Administración por la Universidad Nacional de Trujillo. Magíster en Administración. Profesor Principal y miembro permanente de la Unidad de Investigación de la Facultad de Ciencias Administrativas de la Universidad Nacional Mayor de San Marcos. 
En el año 2003 el Gobierno promulgó la Ley de Promoción y Formalización de la Micro y Pequeña Empresa (Ley № 28015) y su Reglamento (Decreto Supremo № 009-2003-TR), donde nuevamente se establecen dos variables para clasificar una MYPE: número de trabajadores y niveles de ventas anuales.

Así tenemos que el Art. 3o establece que las características concurrentes son:

a) El número total de trabajadores:

La microempresa: abarca de uno (1) hasta diez (10) trabajadores inclusive.

La pequeña empresa: abarca de uno (1) hasta cincuenta (50) trabajadores inclusive.

b) Niveles de ventas:

La microempresa: hasta el monto máximo de 150 Unidades Impositivas Tributarias (UIT).

La pequeña empresa: a partir del monto máximo señalado para las micro empresas y hasta 850 Unidades Impositivas Tributarias (UIT).

Esta caracterización de las Micro y Pequeñas Empresas (MYPEs), basada en el número de trabajadores y nivel de ventas, tiene diferentes interpretaciones. Entidades como el Centro de Promoción de la Pequeña y Micro Empresa (PROMPYME), el Consejo Superior de Contrataciones y Adquisiciones del Estado (CONSUCODE) y el propio Instituto Nacional de Estadística e Informática (INEI), indican que según esta norma los trabajadores independientes son considerados como empresarios de la micro empresa, mientras que el Programa de Estadísticas y Estudios Laborales (PEEL) del Ministerio de Trabajo y Promoción del Empleo (MTPE) considera a los independientes como un rubro diferente y separado de las micro y pequeñas empresas.

El PEEL define a a micro empresa como una unidad económica que ocupa entre 2 y 9 trabajadores, y a la pequeña como la unidad económica que ocupa entre 10 y 49 trabajadores; es decir la unidad económica no es unipersonal.

El estudio de Sánchez y Ferrer, ${ }^{1}$ para calcular la PEA en las MYPEs, incluyó:

- Los dueños de negocios con un máximo de 50 trabajadores.
- Trabajadores dependientes en unidades económicas de menos de 50 trabajadores (excepto actividades extractivas), excluyendo trabajadores del hogar.

- Trabajadores familiares no remunerados.

Otro problema es la "informalidad", término con muchas definiciones y que ha sido asociado a los temas de pobreza, empleo de baja calidad, economía sumergida, ${ }^{2}$ e ilegalidad ${ }^{3}$.

A nivel internacional tenemos también una gama de criterios para identificar las características de las Micro, Pequeñas y Medianas Empresas (MIPYMEs), como se observa en el siguiente cuadro. Países como Francia y México, o instituciones como la Comisión Económica para América Latina y el Caribe (CEPAL) y la Small Business Administration de Estados Unidos, manejan criterios diferentes a lo propuesto por la Ley № 28015.

Cuadro 1. Criterios para clasificar a la Micro, Pequeña y Mediana Empresa

\begin{tabular}{lcl}
\hline Institución & $\begin{array}{c}\text { Tamaño de } \\
\text { la empresa }\end{array}$ & $\begin{array}{c}\text { Número de } \\
\text { trabajadores }\end{array}$ \\
\hline INSEE & $\begin{array}{c}\text { Pequeña } \\
\text { Mediana }\end{array}$ & $\begin{array}{l}\text { De } 50 \text { a } 250 \\
\text { De } 250 \text { a } 1000\end{array}$ \\
FRANCIA & Pequeña & Hasta 250 \\
ADMINISTRATION - & Mediana & De 250 a 500 \\
USA & & \\
Comisión Económica & Pequeña & Entre 5 y 49 \\
para América Latina - & Mediana & De 50 a 250 \\
CEPAL & & \\
EDF-MEXICO & Pequeña & Menos de 25 \\
& Mediana & Entre 50 y 250 \\
Secretaría de & Pequeña & De 16 a 100 \\
Economía & Mediana & De 101 a 250 \\
\hline
\end{tabular}

Fuente: Zorrilla 2002

\section{LAS MYPES EN EL PERÚ}

La Superintendencia Nacional de Administración Tributaria (SUNAT) estimó en el año 2004 que las Micro y Pequeñas Empresas (MYPEs) formales en el Perú son aproximadamente 659,046 (ver Cuadro 2). 
Cuadro 2. Perú: Micro y Pequeñas Empresas 2004

\begin{tabular}{lrr}
\hline \multicolumn{1}{c}{ TIPO EMPRESA } & \multicolumn{1}{c}{$\mathbf{N}^{\mathbf{0}}$} & PORCENTAJE \\
& EMPRESAS & \\
\hline Microempresa & 622,209 & $94.41 \%$ \\
Pequeña empresa & 25,938 & $3.94 \%$ \\
Mediana y Gran Empresa & 10,899 & $1.65 \%$ \\
Total & $\mathbf{6 5 9 , 0 4 6}$ & $\mathbf{1 0 0 . 0 0 \%}$ \\
\hline
\end{tabular}

Fuente: SUNAT-MTPE

Como se puede observar las microempresas representan el $94.41 \%$ del total de empresas y tienen un nivel de ventas brutas anuales menores a 150 UIT, es decir menores a 480, 000 nuevos soles. Si unimos a la micro empresa con la pequeña empresa tenemos que ambas constituyen el $98.35 \%$ de todas las empresas formales a nivel país y tienen ventas anuales menores a $2^{\prime} 720,000$ nuevos soles. Sólo el $1.65 \%$, es decir 10,899 empresas, son medianas o grandes.

Del universo de la MYPE formal, el 77\% tiene un volumen de ventas brutas anuales menores a 13 UIT, es decir menores a 3,500 nuevos soles mensuales o 12,717 dólares americanos anuales que sólo permite la sobrevivencia de una familia; es decir el 77\% de las MYPEs formales se ubican en los estratos de menores ingresos empresariales.

Si analizamos a las MYPEs por su actividad, encontramos que el $82 \%$ se ubica en el sector terciario, de los cuales el $49.6 \%$ se dedican al comercio y el $33.3 \%$ a la atención de servicios. Sólo el $11 \%$ se dedica a actividades de manufac- tura; un $2.9 \%$ se dedica a las actividades agropecuarias y $2.5 \%$ están inmersos en el sector construcción. Las MYPEs que se dedican a la pesca, minería e hidrocarburos alcanzan un total de $0.7 \%$.

La distribución geográfica de las MYPEs formales podemos apreciarla en el Cuadro 3.

Como se observa el $52.4 \%$ de las MYPEs formales se ubican en Lima y Callao y el resto, el $47.6 \%$, en las otras 23 regiones (departamentos). Las regiones más pobres concentran sólo el $1 \%$ de la MYPE, tales como Ayacucho, Tumbes, Moquegua, Amazonas, Pasco, Apurímac, Madre de Dios y Huancavelica.

A nivel macroregional, según datos de SUNAT, la distribución de las MYPEs es la siguiente:

- Macroregión Norte: $19 \%$

- Macroregión Sur: 18\%

- Macroregión Centro: 7\%

- Macroregión Este: 4\%

- Lima-Callao: $52 \%$

Respecto a las MYPEs informales, los datos de SUNAT e INEI son impresionantes:

Cuadro 4. MYPEs formales e informales

\begin{tabular}{lrrr}
\hline & $\begin{array}{c}\text { No de }^{\circ} \\
\text { empresas } \\
\text { formales }\end{array}$ & $\begin{array}{c}\text { No } \\
\text { empresas } \\
\text { informales }\end{array}$ & \multicolumn{1}{c}{$\begin{array}{c}\text { Total de } \\
\text { MYPEs }\end{array}$} \\
\hline Micro & 622,209 & $1^{\prime} 855,075$ & $2^{\prime} 477,284$ \\
Pequeña & 25,938 & 15,395 & 41,333 \\
MYPE & 648,147 & 1870,470 & 2518,617 \\
\hline
\end{tabular}

Fuente: SUNAT e INEI, 2002.

Cuadro 3. Distribución geográfica de las MYPEs formales

\begin{tabular}{lclr}
\hline \multicolumn{1}{c}{ REGIÓN } & TOTAL MYPEs & \multicolumn{1}{c}{ REGIÓN } & TOTAL MYPEs \\
\hline Amazonas & 3,761 & Moquegua & 4,403 \\
Ancash & 18,146 & Puno & 12,189 \\
Cajamarca & 10,625 & Tacna & 11,041 \\
La Libertad & 32,947 & Ayacucho & 5,493 \\
Lambayeque & 21,179 & Huancavelica & 2,266 \\
Piura & 21,931 & Huanuco & 7,526 \\
Tumbes & 4,172 & Junín & 23,415 \\
Apurimac & 3,233 & Lima-Callao & 314,139 \\
Arequipa & 37,793 & Pasco & 4,108 \\
Cusco & 18,379 & Loreto & 9,602 \\
Ica & 16,102 & San Martín & 9,215 \\
Madre de Dios & 2,414 & Ucayali & 7,302 \\
\hline
\end{tabular}


Como puede observarse, las MYPEs formales representan sólo el $25 \%$ del total de empresas a nivel nacional, mientras que las informales son 1'870,470. A nivel de pequeña empresa la mayor cantidad de MYPEs son formales y sólo el $37.2 \%$ son informales.

A nivel de fuerza laboral, las MYPEs tienen las siguientes categorías de empleo:

- Empleadores,

- Asalariados,

- Personas que se declaran como independientes y que dan trabajo a familiares no remunerados, y

- Trabajadores familiares no remunerados.

En el Cuadro 5 se aprecia la composición de la PEA ocupada en la MYPE formal e informal, para el año 2002.

Como se puede observar, más de 7 millones de personas son empleadas en las MYPEs, de las cuales 2'107,297 son trabajadores familiares no remunerados.
La Encuesta Nacional de Hogares 2002-IIIINEI muestra que el 53\% de la Población Económicamente Activa (PEA) ocupada a nivel nacional trabaja en una micro empresa y el $7 \%$ en una pequeña empresa. Las personas que han generado su autoempleo representan el $21 \%$, y las trabajadoras del hogar, el 3\%. En el sector público se encuentran 940 mil trabajadores (aproximadamente el $8 \%$ del total); y el $8 \%$ restante de la PEA se encuentra en la mediana (entre $50 \mathrm{y}$ 199 trabajadores) y gran empresa (más de 200 trabajadores).

En el siguiente cuadro se aprecia que el 86.2\% de la fuerza laboral trabaja en una MYPE informal y sólo el $13.8 \%$ en una MYPE formal. Esto quiere decir que nueve de cada diez personas trabajan en la informalidad. Si observamos únicamente la situación de las micro empresas la realidad es más cruda, ya que el $92.1 \%$ de la fuerza laboral nacional se ubica en este tipo de empresa.

Cuadro 5. MYPEs formales e informales.

\begin{tabular}{|c|c|c|c|c|c|c|c|}
\hline $\begin{array}{c}\text { Categoría } \\
\text { Ocupacional }\end{array}$ & $\begin{array}{c}2 \text { a } 4 \\
\text { trabajadores }\end{array}$ & $\begin{array}{c}5 \text { a } 9 \\
\text { trabajadores }\end{array}$ & $\begin{array}{c}2 \text { a } 9 \\
\text { trabajadores }\end{array}$ & $\begin{array}{c}10 \text { a } 19 \\
\text { trabajadores }\end{array}$ & $\begin{array}{c}20 \text { a } 49 \\
\text { trabajadores }\end{array}$ & $\begin{array}{c}10 \text { a } 49 \\
\text { trabajadores }\end{array}$ & es MYPE \\
\hline TOTAL & 4’963,160 & 1’389,535 & 6’352,695 & 447,915 & 425,772 & 873,687 & $7^{\prime} 226,382$ \\
\hline Empleadores & 454,053 & 131,617 & 585,670 & 32,697 & 8,636 & 41,333 & 627,003 \\
\hline Asalariados & $1 ' 213,141$ & 581,710 & 1'794,851 & 389,883 & 415,734 & 805,617 & $2 ’ 600,468$ \\
\hline TFNR $^{1}$ & 1'587,223 & 493,337 & $2^{\prime} 080,560$ & 25,335 & 1,402 & 26,737 & 2'107,297 \\
\hline \multicolumn{8}{|l|}{ Independiente } \\
\hline con TFNR & $1^{\prime} 708,744$ & 182,870 & $1^{\prime} 891,614$ & 0 & 0 & 0 & 1'891,614 \\
\hline
\end{tabular}

Fuente: INEI, 2002.

Cuadro 6. PEA ocupada en MYPE según grado de formalidad-2002.

\begin{tabular}{lcccccc}
\hline & \multicolumn{2}{c}{$\begin{array}{c}\text { Microempresa (de } \\
\text { 2 a 9 trabajadores) }\end{array}$} & \multicolumn{2}{c}{$\begin{array}{c}\text { Peq. Empresa (de 10 } \\
\text { a } 49 \text { trabajadores) }\end{array}$} & \multicolumn{2}{c}{$\begin{array}{c}\text { MYPE (de 2 a 49 } \\
\text { trabajadores) }\end{array}$} \\
\hline Formal & Absoluto & $\%(\mathrm{mll})$ & Absoluto $\%(\mathrm{mll})$ & Absoluto $\%(\mathrm{mll})$ \\
Informal & 0.5 & $7.9 \%$ & 0.5 & $60 \%$ & 1.0 & $13.8 \%$ \\
TOTAL & 5.8 & $92.1 \%$ & 0.3 & $40 \%$ & 6.2 & $86.2 \%$ \\
& $\mathbf{6 . 3}$ & $\mathbf{1 0 0 . 0 \%}$ & $\mathbf{0 . 8}$ & $\mathbf{1 0 0 \%}$ & $\mathbf{7 . 2}$ & $\mathbf{1 0 0 . 0 \%}$ \\
\hline
\end{tabular}

Fuente: INEI, 2002, elaborado por PEEL. 
En el siguiente cuadro tenemos las MYPEs organizadas por los rubros que más empleo generan:

Cuadro 7. PEA ocupada en MYPE en manufactura por principales rubros - 2002

\begin{tabular}{llc}
\hline CIIU & RAMA & PORCENTAJE \\
\hline 1541 & Elaboración de productos de panadería & $14.0 \%$ \\
3610 & Fabricación de muebles & $12.9 \%$ \\
1810 & Fabricación de prendas de vestir, excepto pieles & $12.9 \%$ \\
1920 & Fabricación de calzado & $5.2 \%$ \\
2811 & Fabricación de productos metálicos para uso estructural & $5.1 \%$ \\
1730 & Fabricación de tejidos de punto y ganchillo & $4.8 \%$ \\
2221 & Actividades de impresión & $3.9 \%$ \\
1552 & Elaboración de vinos & $2.8 \%$ \\
\hline
\end{tabular}

Fuente: ENAHO de INEI, 2002

De acuerdo a la ENAHO-INEI-2002 las personas que conducen unidades económicas con menos de 49 trabajadores son 627,002; siendo el $89.5 \%$ PYMEs informales y sólo el $10.24 \%$ negocios formales.

Un grupo también numeroso de conductores de MYPE son los independientes que utilizan a trabajadores familiares, pero que no son remunerados por desarrollar actividades en dichos emprendimientos.

En la misma encuesta se observa que la gran mayoría de conductores de negocios o micro y pequeñas empresas, son hombres $(79.2 \%)$, mientras que las mujeres representan el $21.8 \%$.

El grado de instrucción de los conductores de las MYPEs formales es significativamente mayor que el de los conductores de MYPEs informales, independientemente de su tamaño. Así tenemos que el $2.6 \%$ de los hombres y el $6.8 \%$ de las mujeres tienen el grado de instrucción de primaria. El $26 \%$ de hombres y el $6 \%$ de mujeres que conducen una MYPE tienen el grado de instrucción secundaria. Una dato interesante es que el $69 \%$ de hombres y el $87 \%$ de mujeres que conducen una MYPE tienen el grado de instrucción superior, sea esta universitaria o técnica y hayan culminado o no su formación superior.

En el aspecto de la edad predominan los conductores, tanto de MYPEs formales como informales, de 25 a 44 años, en especial las unidades más pequeñas de 2 a 4 trabajadores. El tamaño de la MYPE está en correlación positiva con la edad del conductor.

El Ministerio de Trabajo ha hecho un estudio en base a la información de SUNAT y del INEI para estimar el número de MYPE tanto formales como informales con menos de 50 trabajadores, incluyendo trabajadores remunerados y RFNR e independientes; como se observa en el siguiente cuadro:

Cuadro 9. Estimación de MYPEs formales e informales a nivel nacional - 2002.

\begin{tabular}{lr}
\hline & $\begin{array}{r}\mathbf{N}^{\mathbf{0}} \text { de empresas } \\
\text { Totales }\end{array}$ \\
\hline Micro empresa & $\mathbf{2}^{\prime} \mathbf{4 7 7 , 2 8 4}$ \\
Empleadores & 585,670 \\
Independientes con TFNR & $1^{\prime} 891,614$ \\
Pequeña empresa & $\mathbf{4 1 , 3 3 3}$ \\
Empleadores & 41,333 \\
Autoempleo & $\mathbf{2} 512,603$ \\
Independiente calificado & 164,076 \\
Independiente urbano no calificado & $1^{\prime} 708,192$ \\
Independiente rural no calificado & 640,335 \\
TOTAL MYPE & $\mathbf{5}^{\prime} \mathbf{0 3 1 , 2 2 0}$ \\
\hline Fuente: SUNAT, INEI &
\end{tabular}

Cuadro 8. Empleadores de MYPEs formales e informales - 2002.

\begin{tabular}{|c|c|c|c|c|c|c|c|}
\hline & \multicolumn{3}{|c|}{ FORMAL } & \multicolumn{3}{|c|}{ INFORMAL } & \multirow[t]{2}{*}{ TOTAL } \\
\hline & MICRO & PEQUEÑA & MYPE & MICRO & PEQUEÑA & MYPE & \\
\hline HOMBRES & 44,032 & 6,076 & 50,107 & 413,735 & 26,211 & 439,945 & 490,052 \\
\hline MUJERES & 8,958 & 5,178 & 14,136 & 118,946 & 3,868 & 122,814 & 136,950 \\
\hline TOTAL & 52,990 & 11,254 & 64,243 & 532,681 & 30,079 & 562,759 & 627,002 \\
\hline
\end{tabular}

Fuente: ENAHO de INEI, 2002. 
Cuadro 10. Indicadores de ventas MYPE formal - 2004

\begin{tabular}{lcccc}
\hline & Ventas totales & $\begin{array}{c}\text { Participación } \\
\text { ventas MYPE }\end{array}$ & $\begin{array}{c}\text { Número de } \\
\text { empresas }\end{array}$ & $\begin{array}{c}\text { Ventas promedio } \\
\text { por MYPE }\end{array}$ \\
\hline MICRO & $2^{\prime} 324^{\prime} 046,250$ & $50 \%$ & 622,209 & $3,735.2$ \\
PEQUEÑA & $2^{\prime} 291^{\prime} 761,250$ & $50 \%$ & 25,938 & $88,353.6$ \\
MYPE & $4^{\prime} 615^{\prime} 761,250$ & $100 \%$ & 648,147 & \\
\hline
\end{tabular}

Fuente: SUNAT

Del total de MYPEs, 5'031,220, es decir el $57 \%$ son informales (2'865,320 MYPEs) y el $43 \%$ son formales, es decir tenemos 2'165,900 empresas al año 2002.

La información de SUNAT nos permite observar la distribución de las ventas de las MYPEs y darnos cuenta de un desigual comportamiento (Cuadro 10)

Las MYPEs tienen un bajo nivel de ingreso a los mercados externos (ver Cuadro 11), así tenemos que el $0.14 \%$ de las micro empresas logran ventas al exterior, siendo el $2.91 \%$ de la pequeñas empresas. En general el 0.46 MYPE venden en los mercados del mundo y todas son formales para poder declarar exportaciones directas al exterior.

Cuadro 11. MYPES con capacidad exportadora 2004.

\begin{tabular}{lccr}
\hline $\begin{array}{l}\text { Rango de } \\
\text { ventas }\end{array}$ & $\begin{array}{c}\mathbf{N}^{\mathbf{0}} \text { de } \\
\text { empresas } \\
\text { exportadoras } \\
\mathrm{A}\end{array}$ & $\begin{array}{c}\mathbf{N}^{\mathbf{0}} \text { de } \\
\text { empresas } \\
\text { Exportadoras } \\
\mathrm{B}\end{array}$ & $\begin{array}{c}\text { Orientación } \\
\text { exportadora } \\
\text { B/A }\end{array}$ \\
\hline Micro & 622,209 & 918 & 0.14 \\
Pequeña & 25,938 & 756 & 2.91 \\
MYPE & 648,147 & 1,674 & 0.26 \\
Med+Gran & 10,899 & 1,383 & 12.69 \\
TOTAL & $\mathbf{6 5 9 , 0 4 6}$ & $\mathbf{3 , 0 5 7}$ & $\mathbf{0 . 4 6}$ \\
\hline
\end{tabular}

Fuente: SUNAT.
En términos cuantitativos las MYPEs constituyen una buena porción del total de empresas exportadoras, ya que representan el 54.5\% del total de empresas exportadoras; sin embargo según PROMPYME, el monto total de exportaciones para el 2002 ascendió a 133 millones, lo que representa el $1.76 \%$ del valor total de las exportaciones peruanas en dicho año. Otra característica de las MYPEs exportadoras es que no hay sostenibilidad en la capacidad exportadora.

En lo referente al sector económico más del $70 \%$ de las MYPEs exportadoras se ubican en los sectores comercio e industria, y sólo un 7\% en agricultura (Cuadro 12), y principalmente en Lima (véase Cuadro 13).

Cuadro 12. MYPE formal exportadora según sector económico.

\begin{tabular}{lcc}
\hline $\begin{array}{c}\text { Sector } \\
\text { económico }\end{array}$ & $\begin{array}{c}\text { Número de } \\
\text { Empresas }\end{array}$ & Porcentaje \\
\hline Comercio & 718 & $40 \%$ \\
Industria & 622 & $34 \%$ \\
Servicios & 178 & $10 \%$ \\
Agricultura & 118 & $7 \%$ \\
Pesca & 60 & $3 \%$ \\
Otros & 118 & $1 \%$ \\
TOTAL & $\mathbf{1 , 8 1 4}$ & $\mathbf{1 0 0 \%}$ \\
\hline
\end{tabular}

Fuente: Aduanas 2002. Elaboración: PROMPYME.s 
Cuadro 13. MYPE exportadora según ubicación geográfica.

\begin{tabular}{|c|c|c|c|c|}
\hline Departamento & $\begin{array}{c}N^{\circ} \text { de } \\
\text { empresas }\end{array}$ & $\begin{array}{c}\mathrm{N}^{\circ} \text { de } \\
\text { empresas \% }\end{array}$ & $\begin{array}{c}\text { Valor FOB (US\$) } \\
\text { miles \$ }\end{array}$ & $\begin{array}{c}\text { Valor FOB (US\$) } \\
\text { porcentaje }\end{array}$ \\
\hline Ancash & 12 & 0.76 & 960.70 & 0.70 \\
\hline Arequipa & 47 & 2.60 & $4,297.10$ & 3.20 \\
\hline Ayacucho & 5 & 0.30 & $1,282.90$ & 1.00 \\
\hline Cajamarca & 5 & 0.30 & $1,461.90$ & 1.10 \\
\hline Callao & 77 & 4.20 & $3,915.60$ & 2.90 \\
\hline Cusco & 28 & 1.50 & $1,310.50$ & 1.00 \\
\hline Ica & 19 & 1.00 & $2,633.00$ & 2.00 \\
\hline Junín & 14 & 0.80 & 631.50 & 0.50 \\
\hline La Libertad & 21 & 1.20 & 787.50 & 0.60 \\
\hline Lambayeque & 23 & 1.30 & $2,381.00$ & 1.80 \\
\hline Lima & 1,331 & 73.40 & $90,218.00$ & 67.60 \\
\hline Loreto & 37 & 2.00 & $3,061.10$ & 2.30 \\
\hline Madre de Dios & 2 & 0.10 & 709.40 & 0.50 \\
\hline Moquegua & 4 & 0.20 & 479.90 & 0.40 \\
\hline Pasco & 8 & 0.40 & $1,138.80$ & 0.90 \\
\hline Piura & 53 & 2.90 & $3,331.10$ & 2.50 \\
\hline Puno & 10 & 0.60 & 465.70 & 0.30 \\
\hline San Martín & 9 & 0.50 & $1,364.70$ & 1.00 \\
\hline Tacna & 68 & 3.70 & $5,862.30$ & 4.40 \\
\hline Tumbes & 22 & 1.20 & $4,402.40$ & 3.30 \\
\hline Ucayali & 19 & 1.00 & $2,816.30$ & 2.10 \\
\hline TOTAL & 1,814 & 100.00 & $133,511.60$ & 100.0 \\
\hline
\end{tabular}

Fuente: Aduanas, 2002. Elaboración: PROMPYME.

Los principales mercados atendidos por las MYPEs son Estados Unidos, Canadá y la Unión Europea, como se observa en el Cuadro 14.

El acceso al crédito por parte de las MYPEs presenta grandes dificultades. Por ejemplo, las tasas de interés son más altas que los créditos comerciales, tanto en moneda nacional como en moneda extranjera. Así tenemos que la tasa para las MYPEs es mayor en alrededor 40 puntos en la banca múltiple, 23 puntos en las Cajas Rurales, 10 en las Cajas Municipales y de 2 puntos en las EDPYME.

Los bancos que ofertan productos financieros para las MYPEs y que concentran el $80 \%$ de participación son Banco de Crédito, Banco del Trabajo y MiBanco, según datos de diciembre del 2002.

Cuadro 14. MYPEs exportadora según destino.

\begin{tabular}{lcccc}
\hline Destino & $\begin{array}{c}\mathbf{N}^{\mathbf{0}} \text { de } \\
\text { empresas }\end{array}$ & $\begin{array}{c}\text { Participación } \\
\text { porcentual }\end{array}$ & $\begin{array}{c}\text { Valor FOBMiles } \\
\text { US\$ }\end{array}$ & $\begin{array}{c}\text { Participación \% } \\
\text { en ventas }\end{array}$ \\
\hline Canadá & 538 & 29.70 & $20,373.99$ & 15.30 \\
Estados Unidos & 692 & 38.10 & $43,596.43$ & 32.70 \\
Unión Europea & 413 & 22.80 & $23,739.08$ & 17.80 \\
Resto & 171 & 9.40 & $45,802.07$ & 34.30 \\
TOTAL & $\mathbf{1 , 8 1 4}$ & $\mathbf{1 0 0 . 0 0}$ & $\mathbf{1 3 3 , 5 1 1 . 5 8}$ & $\mathbf{1 0 0 . 0 0}$ \\
\hline
\end{tabular}

Fuente: Aduanas. Elaboración: PROMPYME. 
Cuadro 15. Total de crédito a MYPEs - Todas las fuentes (miles de nuevos soles).

\begin{tabular}{lrrrr}
\hline Fuente & 31 mar. 2001 & 31 dic. 2001 & 31 dic. 2002 & 31 dic. 2003 \\
\hline Total banca múltiple & $1,024,046$ & 930,187 & 866,154 & $1,030,062$ \\
Total Cajas Municipales & 343,428 & 427,673 & 593,541 & 832,811 \\
Total Edpymes & 95,637 & 136,304 & 166,645 & 221,087 \\
Total Cajas Rurales & 115,792 & 117,847 & 131,965 & 158,021 \\
TOTAL & $1,578,903$ & $1,612,011$ & $1,758,305$ & $2,242,170$ \\
& & & & \\
\% crecimiento anual & & & $9.08 \%$ & $27.52 \%$ \\
& & & & \\
Total Banca Múltiple & $64.86 \%$ & $57.70 \%$ & $49.26 \%$ & $45.92 \%$ \\
Total Cajas Municipales & $21.75 \%$ & $26.53 \%$ & $33.76 \%$ & $37.14 \%$ \\
Total Edpymes & $6.06 \%$ & $8.46 \%$ & $9.48 \%$ & $9.86 \%$ \\
Total Cajas Rurales & $7.33 \%$ & $7.31 \%$ & $7.51 \%$ & $7.06 \%$ \\
\hline
\end{tabular}

Fuente: SBS.

\section{DESAFÍOS DE LAS MYPE EN EL CONTEXTO DE LA GLOBALIZACIÓN}

\section{Contexto internacional}

El país y el mundo avanzan a un velocidad acelerada hacia la globalización de la economía, la cultura y todas las esferas del quehacer de la humanidad. Esta situación plantea grandes retos a los países y a las MYPEs en cuanto a diversos temas como la generación de empleo, mejora de la competitividad, promoción de las exportaciones y sobre todo el crecimiento del país a tasas mayores al $7 \%$ anual. Esta tasa permitiría que en un periodo de 15 años podamos reducir sustantivamente el 52\% de pobreza, el 20\% de extrema pobreza o las altas tasas de analfabetismo que actualmente tenemos. Ello quiere decir que es posible promover el desarrollo humano en nuestro país, teniendo a las MYPEs como un componente clave.

Para atender adecuadamente al mercado exterior es necesario que las MYPEs, el Estado, los gobiernos regionales y el sector académico concerten un gran proyecto nacional para diversificar la oferta en mercancías tradicionales y no tradicionales, servicios, etc.

El desarrollo de esta propuesta debe tener como base al capital social, es decir un capital humano lleno de salud, instruido, con vivienda digna y servicios de calidad como mínimo, pero también un capital social capaz de construir redes internas y externa que sean proactivas, flexibles y prestas a alinearse con los requerimientos del mundo moderno. Un tercer componente del capital social es provocar una profunda reforma en las estructuras sociales, políticas, culturales, educativas, etc., de nuestro país, en donde las MYPEs deben ser incorporadas.

\section{Contexto nacional}

Si se crean las condiciones básicas propuestas podemos asumir que los resultados positivos de los últimos cuatro años de crecimiento en el país deben servir para un segundo periodo, en donde podamos acercarnos a niveles suficientes para superar el 7\% de crecimiento anual.

Esta posibilidad y las políticas de distribución equitativa de la riqueza sentarán las bases para una nueva perspectiva nacional, dándole a las MYPEs un papel fundamental en el desarrollo nacional, regional y sobre todo local, promoviendo su competitividad y productividad en la perspectiva de mejorar su participación en los mercados internacionales.

La informalidad es un reto que debe ser atendido con firmeza, tanto por las propias MYPEs como por los diversos organismos públicos y privados. Para ello, hasta la fecha, se han aprobado muchos instrumentos como la Ley 28015 y el Plan Nacional Exportador, Plan Nacional de Competitividad, Plan Nacional de Formalización para la Competitividad y Desarrollo de la Micro y Pequeña Empresa, y el Plan Nacional de Ciencia y Tecnología.

\section{Retos de las MYPEs}

a) Heterogeneidad de las MYPEs. Si bien es cierto la Ley 28015 establece las características de las MYPE, todavía son utilizados los criterios 
tradicionales de número de trabajadores y monto de ventas brutas anuales, por lo que necesitamos incorporar otros criterios más técnicos que nos permitan determinar la capacidad de acumulación de capital, además de diferenciar a las MYPE de las unidades económicas que atienden las necesidades de sobrevivencia de las familias. Adicionalmente, es necesario tratar a las MYPEs por su nivel de formalidad o informalidad a partir de considerar los ingresos laborales, aseguramiento de la fuerza laboral, condiciones de empleo y del local de la MYPE. Caracterizar a las MYPEs con estos criterios permitirá establecer estrategias de programas dirigidos a atender una o más de estas características identificadas.

b) Baja productividad. En las MYPEs los niveles de productividad tienen una correlación positiva con el tamaño de la empresa: es decir, a menor tamaño, menor productividad. Las consecuencias de esta baja de productividad repercuten en la baja calidad del empleo, altos niveles de subempleo y los bajos ingresos de los empresarios y trabajadores, como se observa en el siguiente cuadro:

Cuadro 16. Productividad por tamaño de empresa - 1994.

\begin{tabular}{lr}
\hline Tamaño de la empresa & $\begin{array}{r}\text { Productividad } \\
\text { del trabajo (\$) }\end{array}$ \\
\hline De 1 a 4 trabajadores & $4,607.00$ \\
De 5 a 10 trabajadores & $9,774.00$ \\
De 11 a 20 trabajadores & $10,378.00$ \\
De 21 a 50 trabajadores & $15,956.00$ \\
De 51 a 100 trabajadores & $23,147.00$ \\
De 101 a más trabajadores & $51,927.00$ \\
\hline
\end{tabular}

Fuente: CONCYTEC. Mayo, 2003. Lima.

c) La informalidad. Las MYPEs formales representan el 25\% del total de MYPEs, contra los aproximadamente 1.8 millones de MYPEs informales, que alcanzan el $75 \%$ del total de MYPEs del país. A medida que la empresa crece disminuye la informalidad, de allí que las pequeñas empresas formales son 25,938 unidades económicas y las informales son solamente 15,395, según información de la Dirección Nacional de la Micro y Pequeña Empresa del Ministerio de Trabajo y Promoción del Empleo. d) Volumen de ventas en las MYPEs. Los estudios de SUNAT realizados en varios periodos nos indican que también hay una relación directa entre tamaño de empresa y volumen de ventas de éstas. El mayor porcentaje de MYPEs son micro empresas y representan el $77 \%$ de las empresas con ingresos menores a 13 UIT. Además, cuando una MYPE crece se incrementan sus ventas, como se observa en el siguiente cuadro:

Cuadro 17. Distribución porcentual de MYPE formal por rango de ventas en UIT-2004.

\begin{tabular}{lc}
\hline Nivel de ventas & Distribución \% \\
\hline De 0 a 13 UIT & $77 \%$ \\
Más de 13 a 75 UIT & $16 \%$ \\
Más de 75 a 150 UIT & $3 \%$ \\
Más de 150 a 850 UIT & $4 \%$ \\
\hline
\end{tabular}

Fuente: SUNAT.

Elaboración MTPE-DNMYPE.

e) Inserción en el mercado externo. Las MYPEs tienen una débil participación en las exportaciones. Representan sólo el $0.14 \%$ del total de empresas y sus ventas declaradas representan sólo el $0.26 \%$ de participación, según la SUNAT, para el año 2004.

f) Empleo de baja calidad. Como se observa en los cuadros, las MYPEs son las que más empleo generan, sin embargo éste es de baja calidad, sobre todo, en las MYPEs informales, de allí que el nivel de pobreza se incremente en las regiones en las que las MYPEs informales son mayoritarias. Hay una relación positiva directa entre informalidad, empleo informal, niveles bajos de ingreso y pobreza.

En general constatamos que las MYPEs se caracterizan por:

- Limitadas capacidades gerenciales.

- Muy baja productividad.

- Bajos niveles de competitividad.

- Baja rentabilidad.

- Escasa inserción a los mercados, sobre todo al mercado externo.

- Bajo nivel de tecnología.

- Escasa capacidad operativa.

- Limitado acceso a información sobre mercados, tecnología, etc.

- Débil articulación empresarial.

- Barreras para acceder al mercado financiero formal. 
- Barreras burocráticas para acceder a la formalización.

- Poco acceso a los sistemas de desarrollo del capital social y a la capacitación.

Para iniciar un nuevo emprendimiento se presentan una serie de retos que debe superar el emprendedor y que debe significar una propuesta para que estos retos se eliminen o se reduzcan de manera sustantiva:

- Baja relación entre los planes curriculares y las competencias emprendedoras en el sistema educativo nacional, incluyendo a las universidades y las instituciones de formación superior tecnológica.

- Insuficiente apoyo financiero para el inicio de nuevos emprendimientos.

- Alta tasa de mortalidad de las nuevas iniciativas emprendedoras. Se estima que dos de cada tres empresas desaparece en el primer año de operaciones.

- Escaso desarrollo de las competencias gremiales de los líderes de las MYPEs, baja calidad de la representación gremial, poca preocupación por mejorar la competitividad empresarial, visión de corto plazo en el quehacer gremial, y poco apoyo estatal para el fortalecimiento institucional.

- Débil desarrollo de las asociaciones de MYPEs de primer y segundo nivel.

\section{LINEAMIENTOS ESTRATÉGICOS PARA PROMOVER EL DESARROLLO DE LAS MYPES}

\section{Fomento de la productividad y competitividad de las MYPEs}

a) Promover la articulación, asociatividad y cooperación empresarial. Identificar y evaluar las cadenas y conglomerados productivos para fomentar la competitividad $y$ asociatividad de las MYPEs.

b) Facilitar el acceso al mercado en todos los niveles: Es necesario acceder a los mercados local, regional, nacional, así como a la exportación y al mercado estatal, proveyendo la información adecuada y oportuna, además de superar las barreras técnicas y gerenciales.

c) Facilitar el acceso al mercado de servicios de desarrollo empresarial, concertación de programas de servicios dirigido a las MYPEs, sobre todo en lo referido al desarrollo del capital social y alineamiento estratégico con el sistema educativo, haciendo que éste se vincule en perspectiva a las demandas del mercado científico y tecnológico. Promover la acreditación y autoevaluación de las instituciones educativas y de capacitación, públicas y privadas.

d) Fomentar la cultura del emprendimiento en las empresas y en las instituciones educativas públicas y privadas, así como en todas las instituciones públicas que tienen que ver con el sistema integral educativo.

e) Facilitar el acceso a los servicios del sistema financiero y reformar dicho sistema a fin de que llegue a los niveles internacionales de calidad y oferta.

f) Promover una reforma o reingeniería del sistema universitario nacional vinculada con el desarrollo tecnológico y de los procesos de transferencia de tecnología de las instituciones públicas y privadas que generan, adaptan, rescatan y comparten ciencias y tecnología. Promover la investigación aplicada, sobre todo en las universidades públicas y, por consiguiente, otorgarles mayores recursos para transformarlas en CITEs.

g) Desarrollar y desplegar las tecnologías de información y comunicaciones para promover el desarrollo de las MYPEs, en alianza con instituciones públicas y privadas que tienen que ver con el quehacer empresarial, social, jurídico y político.

h) Desarrollar las externalidades adecuadas y suficientes para promover todas las actividades empresariales y las de apoyo e investigación.

i) Fortalecer los gremios MYPEs.

Promoción de la formalización y del mejoramiento de la calidad de empleo y vida

a) Reinventar los sistemas de formalización para las MYPEs, modificando las normas, los procedimientos y las tarifas de manera sustantiva.

b) Establecer un régimen laboral de acuerdo con los niveles de desarrollo empresarial, otorgándole a las MYPEs un régimen especial.

c) Reestructurar el régimen tributario para las MYPEs. 
d) Implementar una estrategia de propiedad intelectual que promueva el uso de esta herramienta por parte de las MYPEs, a través de INDECOPI.

e) Formalización de la propiedad predial.

\section{Reforma del Estado}

a) Diseñar políticas de Estado para promover la competitividad de las MYPEs, que sean eficaces, transparentes y oportunas.

b) Concertar los programas del Estado hacia las MYPEs, erradicando su dispersión.

c) Mejorar la Ley 28015.

\section{Notas}

1 Sánchez y Ferrer (2001).

2 Moser (1978) y OIT (2002).

3 De Soto (1989).

4 TFNR $=$ trabajador familiar no remunerado

\section{BIBLIOGRAFÍA}

Comisión Económica para América Latina (CEPAL) (2003-2004). Estudio Económico de América Latina y el Caribe. CEPAL.

Consejo Nacional de Ciencia y Tecnología (CONCYTEC). Consultoría: Relevamiento e
Identificación de Demandas de Empresas en Innovación Tecnológica. Lima.

De Soto, H. (1989). El otro sendero: la revolución invisible en el tercer mundo. Editorial Sudamericana.

Instituto Nacional de Estadística e Informática (INEI) (2001). Encuesta Nacional de Hogares.

Ministerio de Trabajo y Promoción del Empleo (MTPE) - Dirección Nacional de la Micro y Pequeña Empresa (DNMYPE) (2003). La Informalidad en la Micro y Pequeña Empresa en el Perú.

Moser, C. O. (1978). Informal sector or petty commodoty production: dualism or dependence in urban development? OIT, Resolución relativa al trabajo decente y la economía informal, 2002, págs. 10411064.

Sánchez y Ferrer (2001). Perfil de la PEA ocupada en la Pequeña y Microempresa. En: Boletín de Economía Laboral № 20. Lima, PEELMTPE.

Villarán, Fernando (1998). Riqueza popular, pasión y gloria de la pequeña empresa. Lima, Ediciones del Congreso del Perú, 1998. 